\title{
Mn(III)-Catalyzed Radical Reactions of 1,3-Dicarbonyl Compounds and Cyclopropanols with Vinyl Azides for Divergent Synthesis of Azaheterocycles
}

\author{
Shunsuke Chiba*
}

\begin{abstract}
Mn}(\mathrm{III})$-catalyzed formal [3+2]- and [3+3]-annulations have been developed using readily available vinyl azides with 1,3-dicarbonyl compounds and cyclopropanols. Vinyl azides were successfully applied as a threeatom unit including one nitrogen to prepare various azaheterocycles via $\mathrm{Mn}(\mathrm{III})$-catalyzed radical reactions.
\end{abstract}

Keywords: Azaheterocycles · Cyclopropanols · 1,3-Dicarbonyl compounds · Manganese · Radicals · Vinyl azides

\section{Introduction}

Azaheterocycles are an omnipresent component of numerous natural alkaloids and potent pharmaceutical drugs. ${ }^{[1]}$ Although diverse synthetic approaches toward azaheterocycles have been exploited, ${ }^{[2]}$ there remains a need for conceptually novel and versatile methodologies for chemical synthesis of azaheterocycles from readily available building blocks. Intermolecular annulation reactions allow rapid and selective construction of complex cyclic molecules in a one-pot manner from relatively simple building blocks, which is one of the most ideal processes in organic synthesis from atom-[3] and step-[4] economical point of views. Guided by these views, we have recently been interested in application of vinyl azides as a three-atom unit including one nitrogen to develop new types of annulation reactions for synthesis of azaheterocycles. This review mainly focuses on the $\mathrm{Mn}$ (III)-catalyzed radical

\footnotetext{
${ }^{\star}$ Correspondence: Prof. S. Chiba Nanyang Technological University School of Physical and Mathematical Sciences Division of Chemistry and Biological Chemistry 21 Nanyang Link

Singapore 637371, Singapore

Tel.: +65 65138013

Fax: +65 67911961
}

E-mail: shunsuke@ntu.edu.sg reactions of 1,3-dicarbonyl compounds and cyclopropanols with vinyl azides for a divergent synthesis of azaheterocycles, which have been recently developed in our research group at Nanyang Technological University, Singapore..$^{[5]}$

\section{Backgrounds of Vinyl Azides and Mn(III)-Mediated Oxidative Radical Reactions}

Vinyl azides are readily prepared from commercially available starting materials in several ways, and generally stable/ easy to handle. ${ }^{[6]}$ The application of vinyl azides to synthetic organic reactions have been exclusively relied on their thermal denitrogenative decomposition to vinyl nitrenes, which could be converted into highly reactive strained three-membered ring, $2 \mathrm{H}$-azirines (Scheme 1). ${ }^{[7]}$ Heating of azido cinnamates in aprotic solvents (for example, xylene at $140{ }^{\circ} \mathrm{C}$ ) gave indoles via aromatic $\mathrm{C}-\mathrm{H}$ amination of putative $2 \mathrm{H}$-azirine intermediates, which have been widely utilized for synthesis of indole alkaloids and potent pharmaceutical drugs bearing indole cores. ${ }^{[8,9]}$

We planned to use the $\mathrm{C}=\mathrm{C}$ bond of vinyl azides for initiation of the reactions. Although such attempts have been extremely scarce, we could get two key hints from the following literature precedents to implement our research projects with a radical strategy.

The 1st key: In 1975, Suzuki reported the reaction of $\alpha$-azido styrene with triethylborane, which provided butyrophenone after aqueous workup (Scheme 2). ${ }^{[10]}$ In 1983, Roberts had clarified the reaction mechanism of this $\mathrm{C}-\mathrm{C}$ bond forming reaction, ${ }^{[11]}$ which included a free radical process. An ethyl radical generated from triethylborane with molecular oxygen adds to the $\mathrm{C}=\mathrm{C}$ bond of $\alpha$-azido styrene to give the $\alpha$-azido radical, which undergoes quick denitrogenation to afford an iminyl radical. The reaction of the resulting iminyl radical reacts with triethylborane to generate an ethyl radical that maintains the radical chain. The resulting iminylborane is hydrolyzed under work-up to give butyrophenone.

The 2nd key: Manganese(III)mediated oxidative radical reactions have been extensively studied to construct new $\mathrm{C}-\mathrm{C}$ bonds since the pioneering reactions of acetic acid with alkenes mediated by Mn(III) acetate were reported in 1968. [12] Especially, this Mn(III)-mediated oxidative radical strategy has been applied for the reactions of 1,3-dicarbonyl compounds with various carbon-carbon unsaturated bonds in both inter- and intramolecular manners, leading to highly functionalized

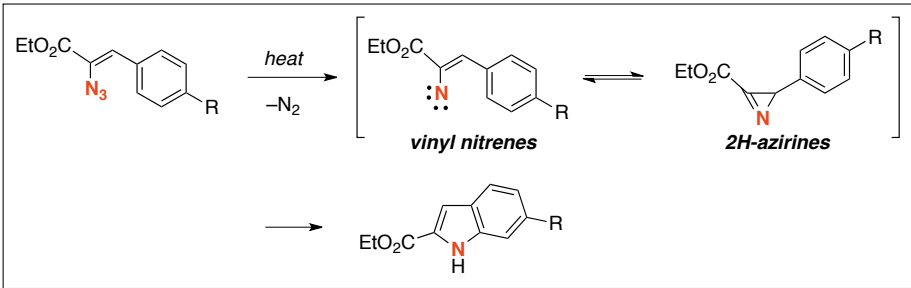

Scheme 1. 


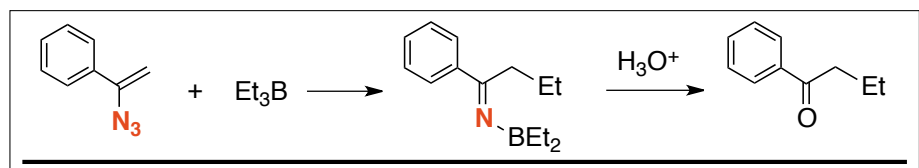

Scheme 2. Reaction of vinyl azides with trialkylboranes via free radical pathway.

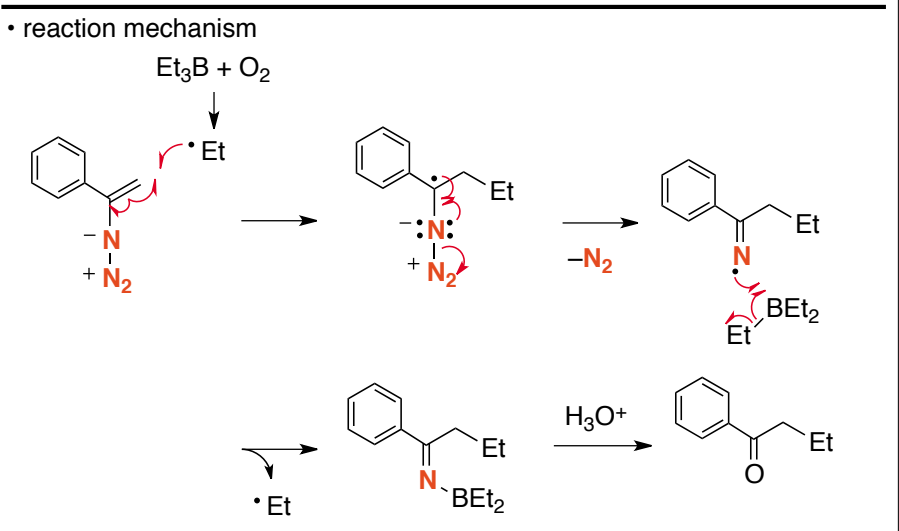

organic molecules (Scheme 3). [13] From a mechanistic point of view, initially formed Mn(III)-enolates undergo rapid loss of $\mathrm{Mn}$ (II) to give $\alpha$-carbonyl radicals, which form a new $\mathrm{C}-\mathrm{C}$ bond with alkenes or alkynes. Similarly, this Mn(III)-mediated oxidative manner can be served for generation of $\beta$-carbonyl radicals from cyclopropanols. ${ }^{[14]}$ However, these strategies basically need superstoichiometric amounts of Mn(III) complexes to complete the reactions because the resulting carbon radicals should be terminated by some oxidative manners (i.e. further oxidation to carbocations) under the reaction conditions.

\section{Design of Mn(III)-Catalyzed Radical Reactions with Vinyl Azides}

We speculated that the combination of the two above-mentioned radical concepts, namely, i) addition of carbon radicals onto vinyl azides and ii) $\mathrm{Mn}$ (III)-mediated oxidative radical reactions using 1,3-dicarbonyl compounds or cyclopropanols, could lead to unique Mn(III)-catalyzed radical reactions (Scheme 4). Our reaction design involves the addition of a carbon radical bearing a carbonyl group to the $\mathrm{C}=\mathrm{C}$ bond of a vinyl azide to provide a new $\mathrm{C}-\mathrm{C}$ bond with generation of an iminyl radical. The iminyl radical then intramolecularly forms a $\mathrm{C}-\mathrm{N}$ bond by the cyclization with the $\mathrm{C}=\mathrm{O}$ bond, leading to various azaheterocycles (Scheme 4 ). This process could potentially be achieved via a redox catalytic manner featured by the two key redox steps: i) oxidative generation of the radical species by the reaction of radical sources with metal oxidant $\left[\mathrm{Mn}^{\mathrm{III}}\right]$ (to be $\left[\mathrm{Mn}^{\mathrm{II}}\right]$ ) (oxidative initiation) and ii) reduction of the resulting iminyl radical by $\left[\mathrm{Mn}^{\mathrm{II}}\right]$ followed by cyclization and protonation to afford azaheterocycles along with regeneration of metal oxidant $\left[\mathrm{Mn}^{\mathrm{II}}\right]$ (reductive termination). This review focuses on this $\mathrm{Mn}(\mathrm{III})$-catalyzed radical reactions of vinyl azides for synthesis of nitrogencontaining heterocycles.

\subsection{Mn(III)-Catalyzed Radical Reactions of Vinyl Azides and 1,3-Dicarbonyl Compounds}

Based on the hypothesis, we have succeeded in developing $\mathrm{Mn}$ (III)-catalyzed reactions of vinyl azides with 1,3-dicarbonyl compounds for synthesis of multi-substituted pyrroles, that could be characterized as a formal [3+2]-annulation process. ${ }^{[15]}$ The reactions of $\alpha$-azido styrene with $\beta$-keto ester, 1,3-diketone, and $\beta$-keto acid using $\mathrm{Mn}(\mathrm{III})$ catalysts are shown in Scheme 5. Interestingly, for each kind of 1,3-dicarbonyl compounds, the preferential $\mathrm{Mn}(\mathrm{III})$-catalyst is varied,$$
\text { a) }
$$$$
\mathrm{O}_{\mathrm{OMe}}^{\mathrm{Mn}(\mathrm{III})}
$$

b)

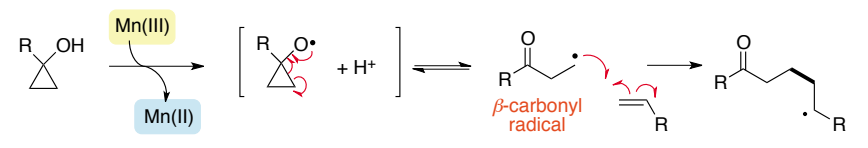

Scheme 3. a) One-electron oxidation of 1,3-dicarbonyls: generation of $\alpha$-carbonyl radical. b) One-electron oxidation of cyclopropanols: Generation of $\beta$-carbonyl radicals.

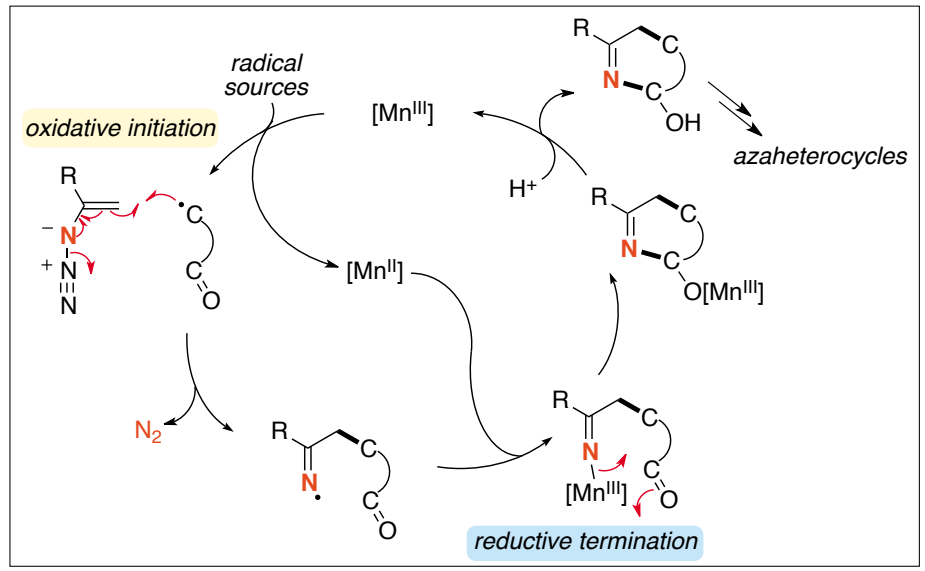

Scheme 4.
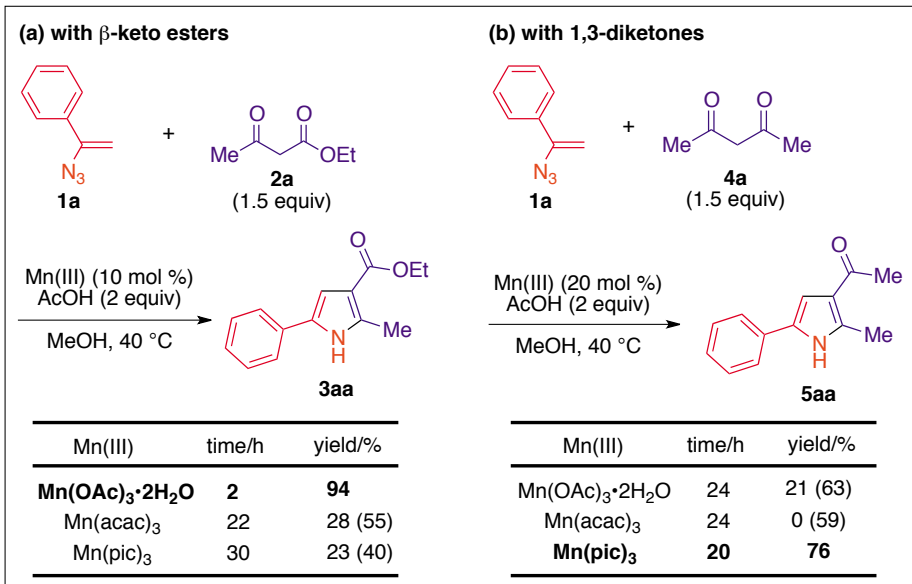

(c) with $\beta$-keto acids
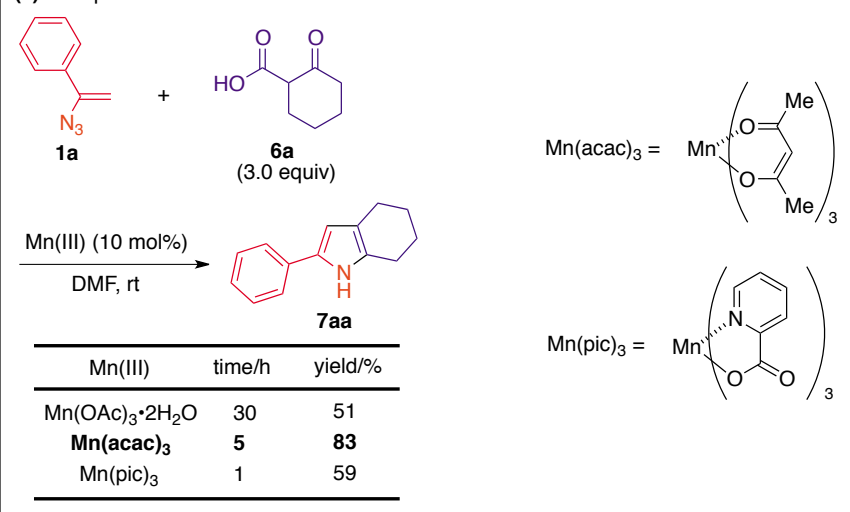


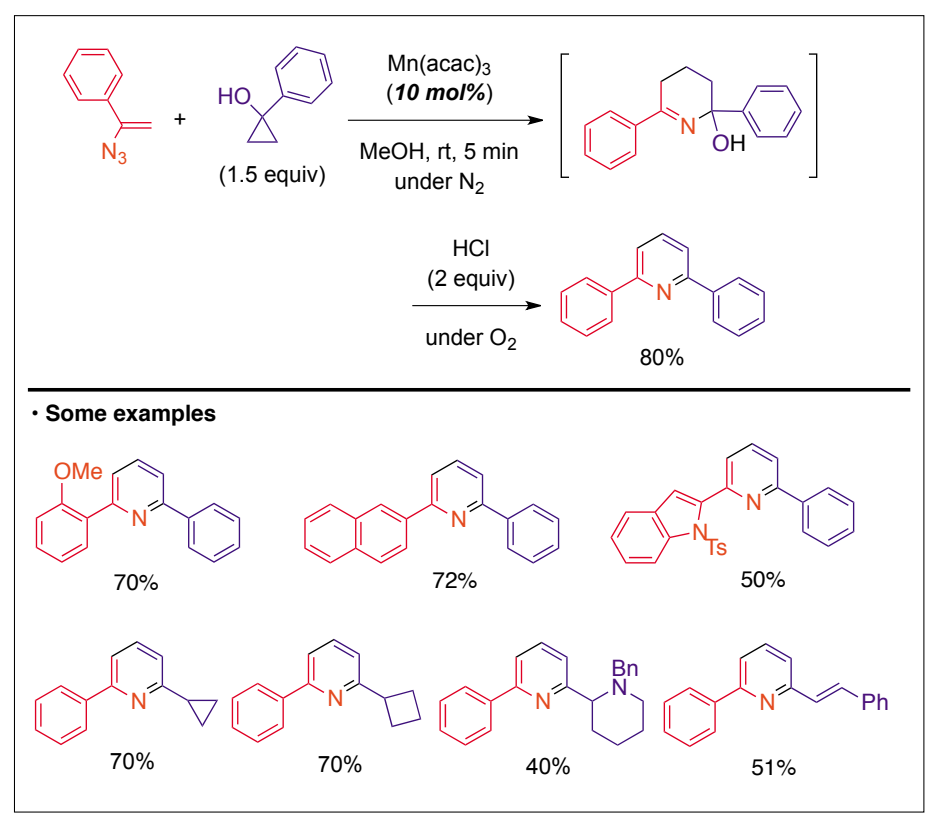

Scheme 6.

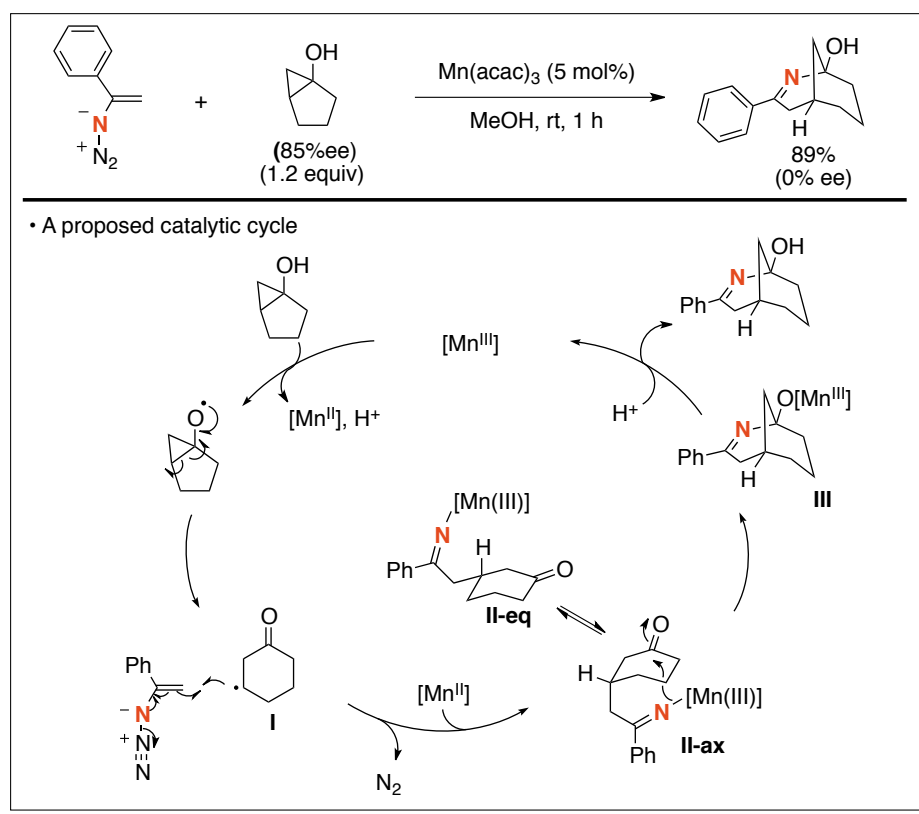

Scheme 7. depending on the nature of 1,3-dicarbonyl compounds as well as the redox potentials of Mn(III) catalysts. ${ }^{[16]}$ As a consequence, $\mathrm{Mn}(\mathrm{OAc})_{3} \cdot 2 \mathrm{H}_{2} \mathrm{O}$ was an effective catalyst for the reactions of vinyl azides and $\beta$-keto esters, while a stronger oxidant, $\mathrm{Mn}(\text { pic })_{3}$, is required for the reactions with 1,3-diketones. On the other hand, $\mathrm{Mn}(\mathrm{acac})_{3}$ was preferred for the reactions of vinyl azides and $\beta$-keto acids. These [3+2]-annulation strategies have a general and wide scope on substituents of both vinyl azides and 1,3-dicarbonyl compounds to give a variety of pyrroles in good yields.

\subsection{Mn(III)-Catalyzed Radical Reactions of Vinyl Azides and Cyclopropanols ${ }^{[17]}$}

We next focused on the use of cyclopropanols as a precursor of $\beta$-carbonyl radicals and investigated their addition reactions toward vinyl azides followed by $\mathrm{C}-\mathrm{N}$ bond formation (formal [3+3]-annulation). The reactions of $\alpha$-azidostyrene and 1-phenylcyclopropanol were investigated to target 2,6-diphenylpyridine
(Scheme 6). It was revealed that treatment of a mixture of vinyl azide and cyclopropanol with a catalytic amount of $\mathrm{Mn}(\mathrm{acac})_{3}$ (10 $\mathrm{mol} \%)$ in $\mathrm{MeOH}$ consumed vinyl azide within $5 \mathrm{~min}$ at room temperature, and the subsequent addition of oxygen $\left(1 \mathrm{~atm} \mathrm{O}_{2}\right.$ as an atmosphere) and $\mathrm{HCl}$ (2 equiv.) provided the desired 2,6-diphenylpyridine in $80 \%$ yield. A wide scope was shown in the substituents of vinyl azides as well as cyclopropanols, and some representative examples were described below.

Next, we envisioned utilizing bicyclic cyclopropanols such as bicyclo[3.1.0] hexan-1-ol as a source of $\beta$-carbonyl radicals (Scheme 7). Interestingly, an unusual 2-azabicyclo[3.3.1]non-2-en-1-ol was isolated in $89 \%$ yield by the reaction with $\alpha$-azidostyrene using only a catalytic amount of $\mathrm{Mn}(\mathrm{acac})_{3}(5 \mathrm{~mol} \%)$. It is noteworthy that treatment of optically active cyclopropanol $(85 \% \quad e e)^{[18]}$ with $\alpha$-azidostyrene afforded the racemic product. No transmission of the chirality of cyclopropanol would suggest that generation of achiral ring-expanded $\beta$-carbonyl radical

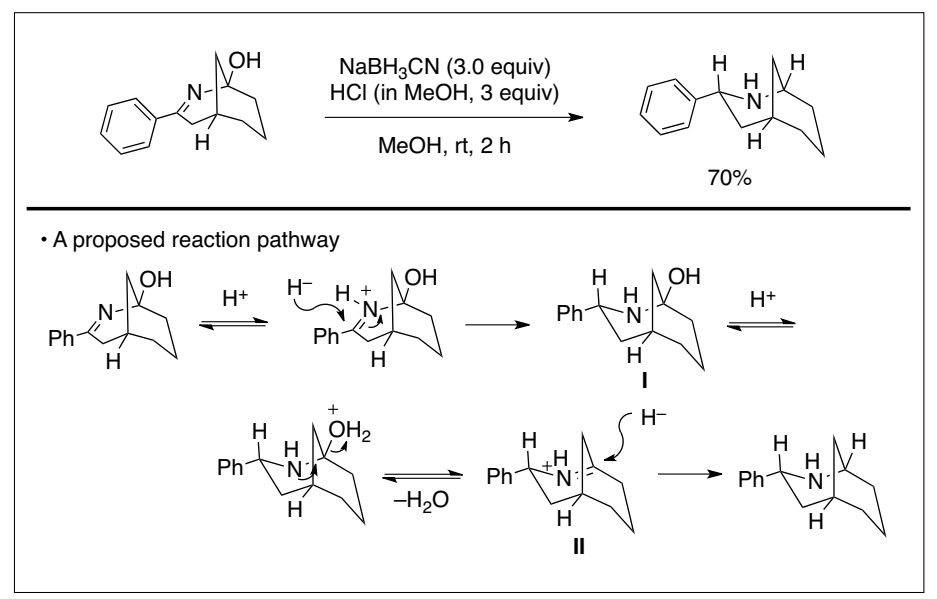

Scheme 8.

[ ${ }^{[19]}$ followed by its radical addition to vinyl azide is involved in the reaction mechanism, forming iminyl manganese(III) II-eq and II- $\boldsymbol{a} \boldsymbol{x}$ placing an imimyl tether in the equatorial- and axial-like position, respectively. Conformational inversion of II-eq to II- $\boldsymbol{a x}$ should be indispensable to achieve the further cyclization of the iminyl manganese II-ax with the carbonyl group to give alkoxy manganese(III) species III that could be protonated to afford 2-azabicyclo[3.3.1]non-2-en-1-ol.

With the preparation method for 2-azabicyclo[3.3.1]non-2-en-1-ol, we then explored their transformations to 2-azabicyclo[3.3.1]nonane (morphan) or 2-azabicyclo[3.3.1]non-2-ene frameworks, which are prevalent in several natural alkaloids as well as biologically active molecules. ${ }^{[20]}$ Treatment of 2-azabicyclo[3.3.1]non-2en-1-ol with $\mathrm{NaBH}_{3} \mathrm{CN}$ in the presence of $\mathrm{HCl}$ induced the double hydride reduction of the $\mathrm{C}=\mathrm{N}$ and $\mathrm{C}-\mathrm{O}$ bonds, affording 2-azabicyclo[3.3.1]nonane stereoselectively in $70 \%$ yield (Scheme 8 ). The first hydride attacked to the $\mathrm{C}=\mathrm{N}$ bond entirely from the less hindered exo-face to form aminal I. Subsequent dehydration of I gave the bridgehead iminium species II, which could be reduced by one more hydride to afford 2-azabicyclo[3.3.1]non2-en-1-ol.

It was found that a one-pot conversion could be achieved starting from the reaction of vinyl azides and cyclopropanols using $\mathrm{Mn}(\mathrm{acac})_{3}$ as a catalyst followed by treatment with $\mathrm{NaBH}_{3} \mathrm{CN}$ (3 equiv.) with $\mathrm{HCl}$ (3 equiv.), producing 2-azabicyclo[3.3.1]nonanes in good yields without isolation of 2-azabicyclo[3.3.1]non-2-en1-ols (Scheme 9). This one-pot/two-step process represented a straightforward procedure for construction of the morphan 


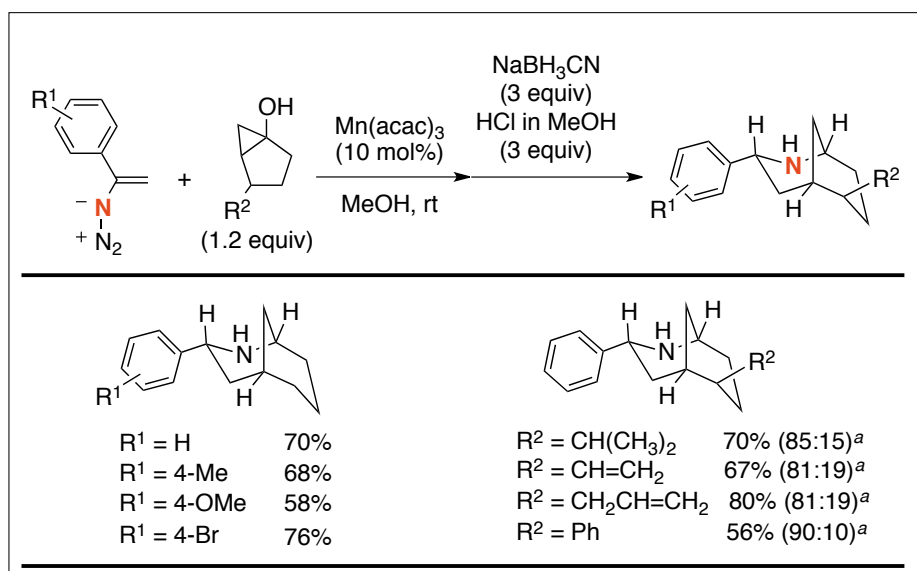

a exo : endo ratio (a major exo product was shown above.)

Scheme 9.

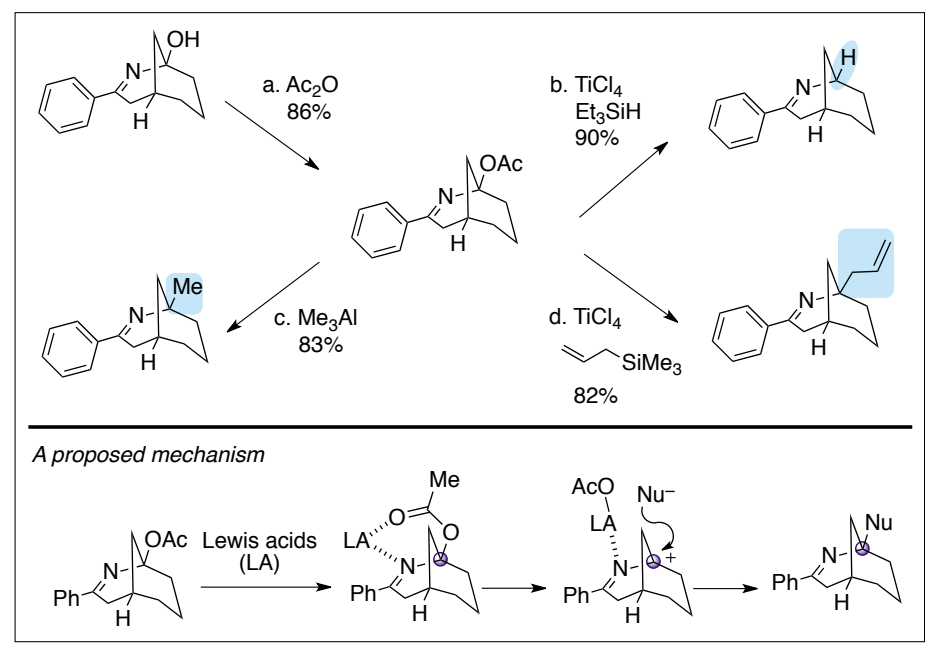

Scheme 10. framework from readily available vinyl azides and bicyclic cyclopropanols.

Further methods for reduction of the $\mathrm{C}-\mathrm{O}$ bond at the bridgehead position were explored using acetate prepared from 2-azabicyclo[3.3.1]non-2-en-1-ol (Scheme 10). Interestingly, $\mathrm{TiCl}_{4}$-mediated reduction of acetate with $\mathrm{Et}_{3} \mathrm{SiH}$ induced selective $\mathrm{C}-\mathrm{O}$ bond cleavage, affording 2-azabicyclo[3.3.1]non-2-ene in $90 \%$ yield keeping the $\mathrm{C}=\mathrm{N}$ bond intact. Similarly, treatment with allyltrimethylsilane- $\mathrm{TiCl}_{4}$ or $\mathrm{Me}_{3} \mathrm{Al}$ provided a new quaternary carbon center at the bridgehead position. These transformations might proceed via a bridgehead carbocation, which was then immediately trapped by present nucleophiles.

Stimulated by the one-pot process developed to prepare 2-azabicyclo[3.3.1] nonanes (Scheme 9), the same procedure was employed to the reaction of $\alpha$-azidostyrenes and bicyclo[4.1.0]heptan- 1-ol to synthesize 2-azabicyclo[4.3.1]decane derivatives. To our delight, 2-azabicyclo[4.3.1]decanes were obtained as a single diastereoisomer via double hydride reductions of the $\mathrm{C}=\mathrm{N}$ and $\mathrm{C}-\mathrm{O}$ bonds of the resulting 2-azabicyclo[4.3.1]dec-2-en-1-ols. Subsequent protection of $\mathrm{N}-\mathrm{H}$ amine with $\mathrm{Cbz}-\mathrm{Cl}$ provided $\mathrm{N}$-benzyloxycarbonyl derivatives in good yields from vinyl azides (Scheme 11).

Melinonine-E (1) was isolated from the bark of Strychnos melinoniana, ${ }^{[21]}$ and its structure was characterized by a unique pentacyclic ring system including indolo[2,3- $a$ ]quinolizidine and morphan frameworks. ${ }^{[22]}$ The first synthesis of $( \pm)$-melinonine-E was accomplished by Bonjoch. ${ }^{[23]}$ We envisioned that the 2-azabicyclo[3.3.1]nonane moiety of melinonine-E would be constructed by $\mathrm{Mn}$ (III)mediated $[3+3]$-annulation of $\alpha$-indolyl vinyl azide $\mathbf{2}$ and bicyclic cyclopropanol $\mathbf{3}$

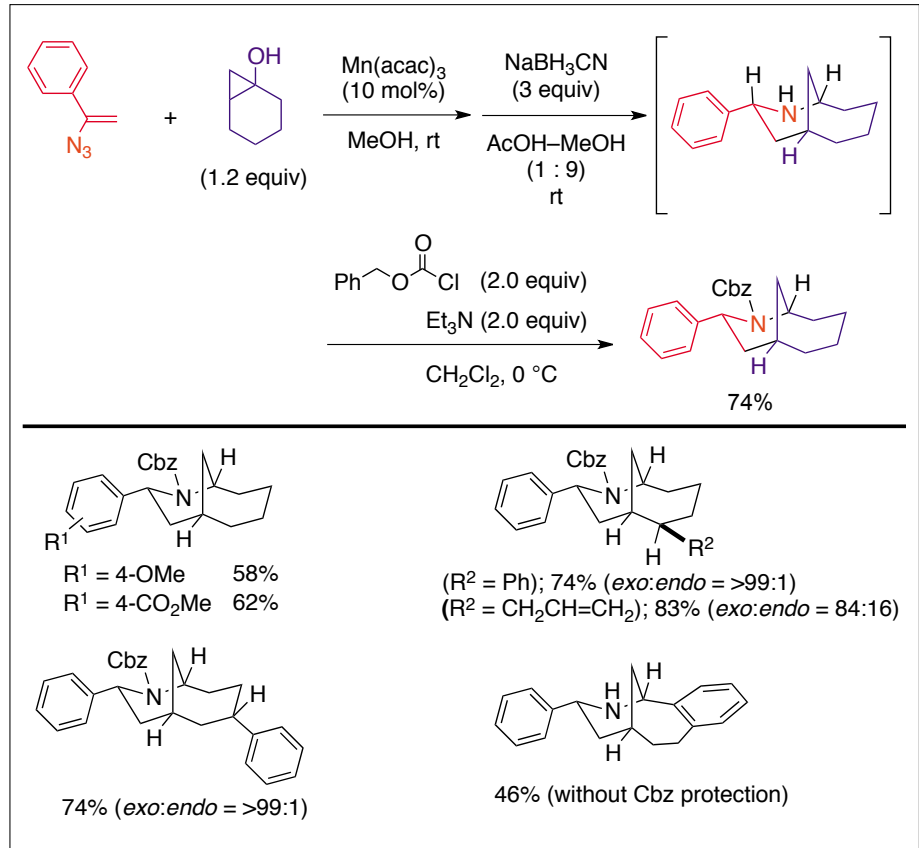

Scheme 11. bearing a hydroxymethyl tether followed by reduction of the $\mathrm{C}=\mathrm{N}$ and bridgehead $\mathrm{C}-\mathrm{O}$ bonds. [3+3]-Annulation of $\alpha$-indolylvinyl azide $\mathbf{2}$ and bicyclic cyclopropanol $\mathbf{3}$ afforded azabicyclic compound $\mathbf{4}$ in $88 \%$ yield in a $2 \mathrm{~g}$ scale in a diastereoselective manner (exo:endo $=85: 15$ ), although it needed 1.6 equiv. of $\mathrm{Mn}(\mathrm{acac})_{3}$ to complete the reaction (Scheme 12). After conversion of 4 into its acetate, the bridgehead $\mathrm{C}-\mathrm{O}$ bond was reduced by the $\mathrm{Et}_{3} \mathrm{SiH}-\mathrm{TiCl}_{4}$ protocol to afford cyclic imine 5. Subsequent reduction of the $\mathrm{C}=\mathrm{N}$ bond of $\mathbf{5}$ with $\mathrm{AlH}_{3}$ prepared from $\mathrm{LiAlH}_{4}-\mathrm{AlCl}_{3}$, leading to not only the entire reduction of imine and $N$-tosyl moieties but also partial deprotection of the TBDPS group. Reductive $N$-alkylation of the resulting $N-\mathrm{H}$ amines of 6 with dimethoxyacetaldehyde in the presence of $\mathrm{NaBH}(\mathrm{OAc})_{3}$ provided 7 and 8 in $43 \%$ and $12 \%$ yield, respectively. The remaining TBDPS group of 7 was removed with $n$ - $\mathrm{Bu}_{4} \mathrm{NF} . \mathrm{BBr}_{3}$-induced cyclization of $\mathbf{8}$ proceeded cleanly to afford the cyclic alcohol 9, which further underwent dehydration with maleic acid in water followed by dehydrogenation with palladium black in one-pot manner, affording ( \pm )-melinonine-E (1) as a perchlorate salt in $44 \%$ yield from 8 . The ${ }^{1} \mathrm{H}$ and ${ }^{13} \mathrm{C} N M R$ data of the synthetic $( \pm)$-melinonine-E perchlorate were identical to those of the reported ones.

\section{Conclusions}

We have developed a divergent synthetic route to construct azaheterocycles from readily available vinyl azides and 1,3-dicarbonyl compounds/cyclopropanols via formal [3+2]- or [3+3]-annulation using Mn(III) complexes. A series of azaheterocycles such as pyrroles, pyridines, and azabicyclic compounds have been successfully prepared by utilizing this promising strategy. Further investigation to explore 


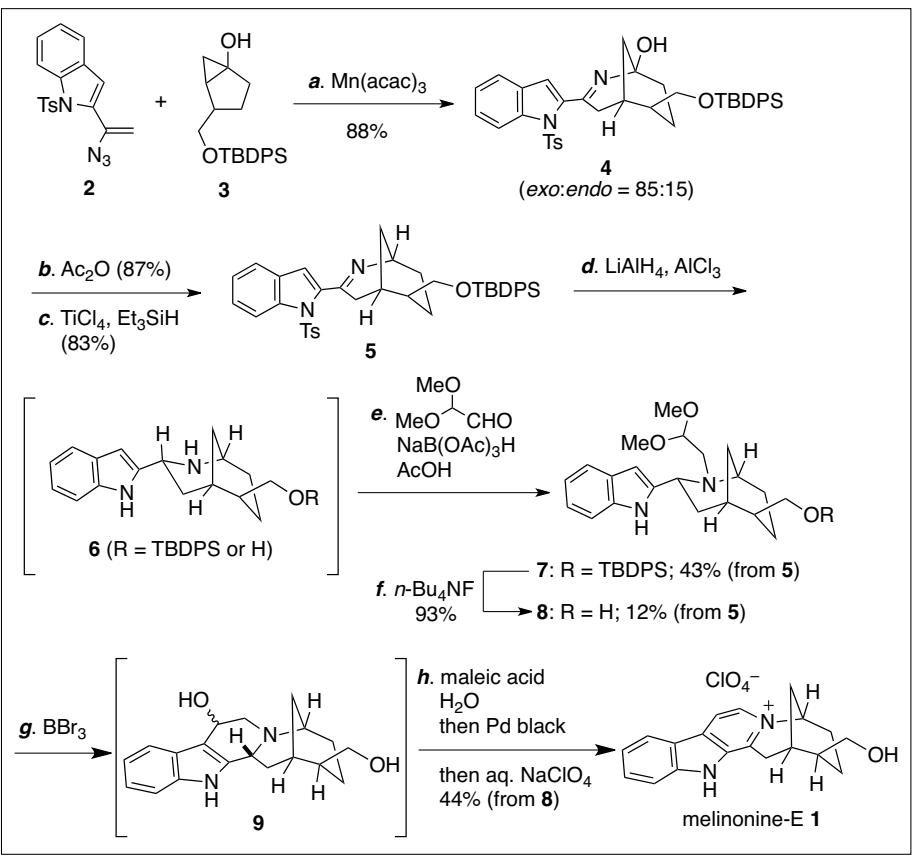

Scheme 12. ${ }^{a}$ Reagents and conditions: (a) 3 (3.0 equiv, added by a syringe pump), $\mathrm{Mn}(\mathrm{acac})_{3}$ (1.6 equiv), $\mathrm{MeOH}, \mathrm{rt}, 8 \mathrm{~h}, 88 \%$; (b) $\mathrm{Ac}_{2} \mathrm{O}(8.0$ equiv), $\mathrm{Et}_{3} \mathrm{~N}$ (2.0 equiv), DMAP (0.1 equiv), $\mathrm{CH}_{2} \mathrm{Cl}_{2}, \mathrm{rt}, 12 \mathrm{~h}, 87 \%$; (c) $\mathrm{TiCl}_{4}$ (1.5 equiv), $\mathrm{Et}_{3} \mathrm{SiH}$ (2.0 equiv), $\mathrm{CH}_{2} \mathrm{Cl}_{2}, \mathrm{rt}, 4 \mathrm{~h}, 83 \%$; (d) $\mathrm{AlCl}_{3}$ (5.0 equiv), $\mathrm{LiAlH}_{4}$ (15.0 equiv), rt, $30 \mathrm{~h}$; (e) (MeO) ${ }_{2} \mathrm{CHCHO}$ (1.5 equiv), $\mathrm{NaB}(\mathrm{OAc})_{3} \mathrm{H}$ (1.5 equiv), $\mathrm{CH}_{2} \mathrm{Cl}_{2}, 0^{\circ} \mathrm{C}, 30 \mathrm{~min}, 7$ (43\% from 5 ) +8 (12\% from 5); (f) $n$ $\mathrm{Bu}_{4} \mathrm{NF}$ (1.5 equiv), THF, rt, $36 \mathrm{~h}, 93 \%$; (g) $\mathrm{BBr}_{3}$ (8.0 equiv), $\mathrm{CH}_{2} \mathrm{Cl}_{2},-78{ }^{\circ} \mathrm{C}$, $3 \mathrm{~h}$; (h) maleic acid (6.0 equiv), $\mathrm{H}_{2} \mathrm{O}$, rt, overnight, then Pd black (excess), reflux, 50 h; aqueous $\mathrm{NaClO}_{4}$ (3.0 equiv), rt, $44 \%$ (from 8 ).

other modes of annulation reactions of vinyl azides to prepare azaheterocycles is currently underway.

\section{Acknowledgment}

This work was supported by funding from Nanyang Technological University, Singapore Ministry of Education, and Science \& Engineering Research Council (A*STAR grant No. 082101 0019). We thank Dr. Yongxin $\mathrm{Li}$ (Division of Chemistry and Biological Chemistry, School of Physical and Mathematical Sciences, Nanyang Technological University) for assistance in $\mathrm{X}$-ray crystallographic analysis.

Received: March 9, 2012

[1] For recent reviews, see: a) G. L. Thomas, C. W. Johannes, Curr. Opin. Chem. Biol. 2011, 15, 516; b) R. Tohme, N. Darwiche, H. GaliMuhtasib, Molecules 2011, 16, 9665; c) S. Dandapani, L. A. Marcaurelle, Curr. Opin. Chem. Biol. 2010, 14, 362; d) M. E. Welsch, S.
A. Snyder, B. R. Stockwell, Curr Opin. Chem. Biol. 2010, 14, 347; e) J. S. Carey, D. Laffan, C. Thomson, M. T. Williams, Org. Biomol. Chem. 2006, 4, 2337.

[2] a) J. A. Joule, K. Mills, 'Heterocyclic Chemistry', 5th edition, Wiley-Blackwell, 2010; b) 'Progress in Heterocyclic Chemistry', Eds. G. W. Gribble, J. A. Joule, Elsevier: Oxford, 2008; Vol. 20 and others in this series; c) 'Comprehensive Heterocyclic Chemistry III', Eds. A. R. Katritzky, C. A. Ramsden, E. F. V. Scriven, R. J. K. Taylor, Pergamon: Oxford, 2008; d) T. Eicher, S. Hauptmann, The Chemistry of Heterocycles; Wiley-VCH: Weinheim, 2003.

[3] a) B. M. Trost, Angew. Chem. Int. Ed. 1995, 34, 259; b) B. M. Trost, Science 1991, 254, 1471.

[4] a) P. A. Wender, V. A. Verma, T. J. Paxton, T. H. Pillow, Acc. Chem. Res. 2008, 41, 40. b) P. A. Wender, M. P. Croatt, B. Witulski, Tetrahedron 2006, 62, 7505 .

[5] For a prior account on our azide chemistry, see S. Chiba, Synlett 2012, 23, 21

[6] For a review, see: K. Banert, in 'Organic Azides: Syntheses and Applications', Eds. S. Bräse, K. Banert, Wiley, 2010; p. 115.
[7] For our recent works on heterocycle synthesis using $2 \mathrm{H}$-azirines generated from vinyl azides, see: a) E. P. J. Ng, Y.-F. Wang, B. W.-Q. Hui, G. Lapointe, S. Chiba, Tetrahedron 2011, 67, 7728. b) S. Chiba, Y.-F. Wang, G. Lapointe, K Narasaka, Org. Lett. 2008, 10, 313.

[8] a) C. J. Moody, in 'Comprehensive Organic Synthesis', Eds. B. M. Trost, I. Fleming, S. Ley, Pergamon: Oxford, 1991; Vol. 7, p 21; b) G. Smolinsky, C. A. Pryde, J. Org. Chem. 1968 33, 2411; c) G. L'abbe, Angew. Chem., Int. Ed. Engl. 1975, 14, 775; d) H. Hemetsberger, D. Knittle, H. Weidmann, Monatsh. Chem. 1970 , 101, 161; e) A. R. MacKenzie, C. J. Moody, C. W. Rees, J. Chem. Soc., Chem. Commun. 1983 , 1372; f) R. E. Bolton, C. J. Moody, M. Pass, C. W. Rees, G. Tojo, J. Chem. Soc., Perkin Trans. 1 1988, 2491.

[9] $\mathrm{Fe}(\mathrm{II})$ - and $\mathrm{Rh}$ (II)-catalyzed indole formation from azidocinnamates was reported, see: a) J. Bonnamour, C. Bolm, Org. Lett. 2011, 13, 2012; b) B. J. Stokes, H. Dong, B. E. Lesile, A. L. Pumphrey, T. G. Driver, J. Am. Chem. Soc. 2007, 129, 7500 .

[10] A. Suzuki, Tetrahedron Lett. 1975, 2195.

[11] B. P. Roberts, Tetrahedron Lett. 1983, 24, 3779.

[12] a) J. B. Bush, Jr., H. Finkbeiner, J. Am. Chem Soc. 1968, 90, 5903; b) E. I. Heiba, R. M. Sessau, W. J. Koehl, Jr. J. Am. Chem. Soc. 1968, 90, 5905 .

[13] For selected reviews, see: a) B. B. Snider, Chem. Rev. 1996, 96, 339; b) B. B. Snider, in 'Radicals in Organic Synthesis', Vol. 1, Eds. P. Renaud, M. P. Sibi, Wiley-VCH, Weinheim, 2001, p. 198

[14] a) N. Iwasawa, S. Hayakawa, M. Funahashi, K. Isobe, K. Narasaka, Bull. Chem. Soc. Jpn. 1993, 66,$819 ;$ b) N. Iwasawa, S. Hayakawa, K. Isobe, K. Narasaka, Chem. Lett. 1991, 1193.

[15] a) E. P. J. Ng, Y.-F. Wang, S. Chiba, Synlett 2011, 783; b) Y.-F. Wang, K. K. Toh, S. Chiba, K. Narasaka, Org. Lett. 2008, 10, 5019.

[16] S. A. Richert, P. K. S. Tsang, D. T. Sawyer, Inorg. Chem. 1988, 27, 1814

[17] a) Y.-F. Wang, K. K. Toh, E. P. J. Ng, S. Chiba, J. Am. Chem. Soc. 2011, 133, 6411; b) Y.-F. Wang, S. Chiba, J. Am. Chem. Soc. 2009, 133 12570

[18] H. Du, J. Long, Y. Shi, Org. Lett. 2006, 8, 2827.

[19] For discussion on racemization of cyclohexy radicals, see: a) A. J. Buckmelter, A. I. Kim, S. D. Rychnovsky, J. Am. Chem. Soc. 2000, 122, 9386; b) B. P. Roberts, A. J. Steel, J. Chem. Soc., Perkin Trans. II 1992, 2025, and references therein.

[20] For a review on synthesis of 2-azabicyclo[3.3.1] nonanes, see: J. Bonjoch, F. Diaba, B. Bradshaw, Synthesis 2011, 993.

[21] E. Bächli, C. Vamvacas, H. Schmid, P. Karrer, Helv. Chim. Acta 1957, 40, 1167.

[22] R. P. Borris, A. Guggisberg, M. Hesse, Helv Chim. Acta 1984, 67, 455.

[23] a) J. Quirante, C. Escolano, A. Merino, J. Bonjoch, J. Org. Chem. 1998, 63, 968; b) J. Quirante, C. Escolano, J. Bosch, J. Bonjoch, J. Chem. Soc., Chem. Commun. 1995, 2141. 